\title{
On Grids in Point-Line Arrangements in the Plane
}

\author{
Mozhgan Mirzaei \\ Department of Mathematics, University of California at San Diego, La Jolla, CA, 92093 USA \\ momirzae@ucsd.edu
}

\section{Andrew Suk}

Department of Mathematics, University of California at San Diego, La Jolla, CA, 92093 USA

asuk@ucsd.edu

\begin{abstract}
The famous Szemerédi-Trotter theorem states that any arrangement of $n$ points and $n$ lines in the plane determines $O\left(n^{4 / 3}\right)$ incidences, and this bound is tight. In this paper, we prove the following Turán-type result for point-line incidence. Let $\mathcal{L}_{a}$ and $\mathcal{L}_{b}$ be two sets of $t$ lines in the plane and let $P=\left\{\ell_{a} \cap \ell_{b}: \ell_{a} \in \mathcal{L}_{a}, \ell_{b} \in \mathcal{L}_{b}\right\}$ be the set of intersection points between $\mathcal{L}_{a}$ and $\mathcal{L}_{b}$. We say that $\left(P, \mathcal{L}_{a} \cup \mathcal{L}_{b}\right)$ forms a natural $t \times t$ grid if $|P|=t^{2}$, and $\operatorname{conv}(P)$ does not contain the intersection point of some two lines in $\mathcal{L}_{a}$ and does not contain the intersection point of some two lines in $\mathcal{L}_{b}$. For fixed $t>1$, we show that any arrangement of $n$ points and $n$ lines in the plane that does not contain a natural $t \times t$ grid determines $O\left(n^{\frac{4}{3}-\varepsilon}\right)$ incidences, where $\varepsilon=\varepsilon(t)>0$. We also provide a construction of $n$ points and $n$ lines in the plane that does not contain a natural $2 \times 2$ grid and determines at least $\Omega\left(n^{1+\frac{1}{14}}\right)$ incidences.
\end{abstract}

2012 ACM Subject Classification Mathematics of computing $\rightarrow$ Combinatoric problems

Keywords and phrases Szemerédi-Trotter Theorem, Grids, Sidon sets

Digital Object Identifier 10.4230/LIPIcs.SoCG.2019.50

Funding Mozhgan Mirzaei: Supported by NSF grant DMS-1800746.

Andrew Suk: Supported by an NSF CAREER award and an Alfred Sloan Fellowship.

\section{Introduction}

Given a finite set $P$ of points in the plane and a finite set $\mathcal{L}$ of lines in the plane, let $I(P, \mathcal{L})=\{(p, \ell) \in P \times \mathcal{L}: p \in \ell\}$ be the set of incidences between $P$ and $\mathcal{L}$. The incidence graph of $(P, \mathcal{L})$ is the bipartite graph $G=(P \cup \mathcal{L}, I)$, with vertex parts $P$ and $\mathcal{L}$, and $E(G)=I(P, \mathcal{L})$. If $|P|=m$ and $|\mathcal{L}|=n$, then the celebrated theorem of Szemerédi and Trotter $[16]$ states that

$$
|I(P, \mathcal{L})| \leq O\left(m^{2 / 3} n^{2 / 3}+m+n\right) .
$$

Moreover, this bound is tight which can be seen by taking the $\sqrt{m} \times \sqrt{m}$ integer lattice and bundles of parallel "rich" lines (see [13]). It is widely believed that the extremal configurations maximizing the number of incidences between $m$ points and $n$ lines in the plane exhibit some kind of lattice structure. The main goal of this paper is to show that such extremal configurations must contain large natural grids.

Let $P$ and $P_{0}$ (respectively, $\mathcal{L}$ and $\mathcal{L}_{0}$ ) be two sets of points (respectively, lines) in the plane. We say that the pairs $(P, \mathcal{L})$ and $\left(P_{0}, \mathcal{L}_{0}\right)$ are isomorphic if their incidence graphs are isomorphic. Solymosi made the following conjecture (see page 291 in [2]).

Conjecture 1.1. For any set of points $P_{0}$ and for any set of lines $\mathcal{L}_{0}$ in the plane, the maximum number of incidences between $n$ points and $n$ lines in the plane containing no subconfiguration isomorphic to $\left(P_{0}, \mathcal{L}_{0}\right)$ is o( $\left.n^{\frac{4}{3}}\right)$.

(c) (i) $\odot$ Mozhgan Mirzaei and Andrew Suk;

c. licensed under Creative Commons License CC-BY

35th International Symposium on Computational Geometry (SoCG 2019).

Editors: Gill Barequet and Yusu Wang; Article No. 50; pp. 50:1-50:11

Leibniz International Proceedings in Informatics

LI I ICS Schloss Dagstuhl - Leibniz-Zentrum für Informatik, Dagstuhl Publishing, Germany 


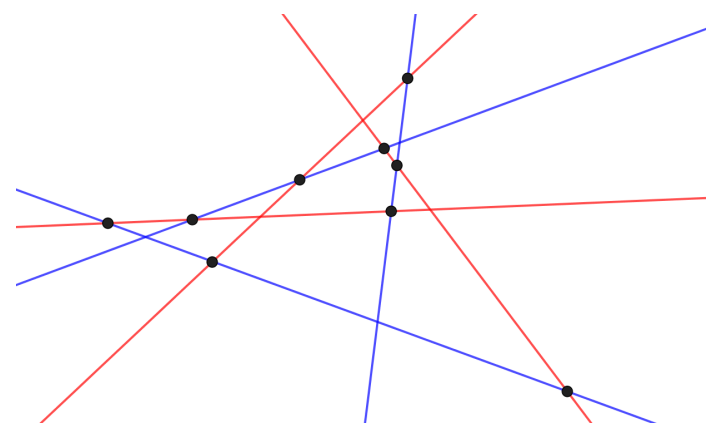

Figure 1 An example with $\left|\mathcal{L}_{a}\right|=\left|\mathcal{L}_{b}\right|=3$ and $|P|=9$ in Theorem 1.3.

In [15], Solymosi proved this conjecture in the special case that $P_{0}$ is a fixed set of points in the plane, no three of which are on a line, and $\mathcal{L}_{0}$ consists of all of their connecting lines. However, it is not known if such configurations satisfy the following stronger conjecture.

- Conjecture 1.2. For any set of points $P_{0}$ and for any set of lines $\mathcal{L}_{0}$ in the plane, there is a constant $\varepsilon=\varepsilon\left(P_{0}, \mathcal{L}_{0}\right)$, such that the maximum number of incidences between $n$ points and $n$ lines in the plane containing no subconfiguration isomorphic to $\left(P_{0}, \mathcal{L}_{0}\right)$ is $O\left(n^{4 / 3-\varepsilon}\right)$.

Our first theorem is the following.

Theorem 1.3. For fixed $t>1$, let $\mathcal{L}_{a}$ and $\mathcal{L}_{b}$ be two sets of $t$ lines in the plane, and let $P_{0}=\left\{\ell_{a} \cap \ell_{b}: \ell_{a} \in \mathcal{L}_{a}, \ell_{b} \in \mathcal{L}_{b}\right\}$ such that $\left|P_{0}\right|=t^{2}$. Then there is a constant $c=c(t)$ such that any arrangement of $m$ points and $n$ lines in the plane that does not contain $a$ subconfiguration isomorphic to $\left(P_{0}, \mathcal{L}_{a} \cup \mathcal{L}_{b}\right)$ determines at most $c\left(m^{\frac{2 t-2}{3 t-2}} n^{\frac{2 t-1}{3 t-2}}+m^{1+\frac{1}{6 t-3}}+n\right)$ incidences.

See the Figure 1. As an immediate corollary, we prove Conjecture 1.2 in the following special case.

- Corollary 1.4. For fixed $t>1$, let $\mathcal{L}_{a}$ and $\mathcal{L}_{b}$ be two sets of $t$ lines in the plane, and let $P_{0}=\left\{\ell_{a} \cap \ell_{b}: \ell_{a} \in \mathcal{L}_{a}, \ell_{b} \in \mathcal{L}_{b}\right\}$. If $\left|P_{0}\right|=t^{2}$, then any arrangement of $n$ points and $n$ lines in the plane that does not contain a subconfiguration isomorphic to $\left(P_{0}, \mathcal{L}_{a} \cup \mathcal{L}_{b}\right)$ determines at most $O\left(n^{\frac{4}{3}-\frac{1}{9 t-6}}\right)$ incidences.

In the other direction, we prove the following.

- Theorem 1.5. Let $\mathcal{L}_{a}$ and $\mathcal{L}_{b}$ be two sets of 2 lines in the plane, and let $P_{0}=\left\{\ell_{a} \cap \ell_{b}\right.$ : $\left.\ell_{1} \in \mathcal{L}_{a}, \ell_{b} \in \mathcal{L}_{b}\right\}$ such that $\left|P_{0}\right|=4$. For $n>1$, there exists an arrangement of $n$ points and $n$ lines in the plane that does not contain a subconfiguration isomorphic to $\left(P_{0}, \mathcal{L}_{a} \cup \mathcal{L}_{b}\right)$, and determines at least $\Omega\left(n^{1+\frac{1}{14}}\right)$ incidences.

Given two sets $\mathcal{L}_{a}$ and $\mathcal{L}_{b}$ of $t$ lines in the plane, and the point set $P_{0}=\left\{\ell_{a} \cap \ell_{b}: \ell_{a} \in\right.$ $\left.\mathcal{L}_{a}, \ell_{b} \in \mathcal{L}_{b}\right\}$, we say that $\left(P_{0}, \mathcal{L}_{a} \cup \mathcal{L}_{b}\right)$ forms a natural $t \times t$ grid if $\left|P_{0}\right|=t^{2}$, and the convex hull of $P_{0}, \operatorname{conv}\left(P_{0}\right)$, does not contain the intersection point of any two lines in $\mathcal{L}_{a}$ and does not contain the intersection point of any two lines in $\mathcal{L}_{b}$. See Figure 2.

Theorem 1.6. For fixed $t>1$, there is a constant $\varepsilon=\varepsilon(t)$, such that any arrangement of $n$ points and $n$ lines in the plane that does not contain a natural $t \times t$ grid determines at most $O\left(n^{\frac{4}{3}-\varepsilon}\right)$ incidences. 


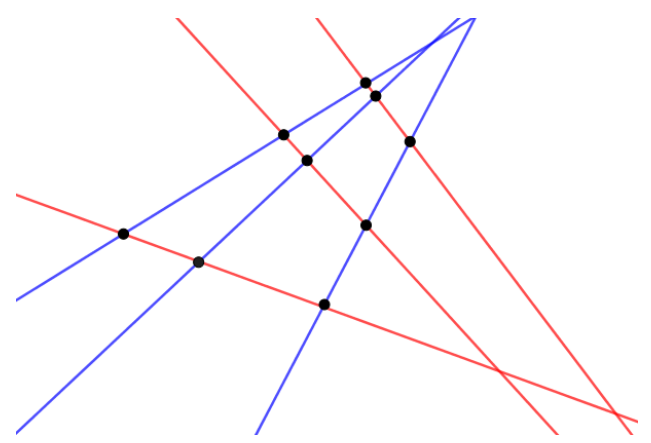

Figure 2 An example of a natural $3 \times 3$ grid.

Let us remark that $\varepsilon=\Omega\left(1 / t^{2}\right)$ in Theorem 1.6 , and can be easily generalized to the off-balanced setting of $m$ points and $n$ lines.

We systemically omit floor and ceiling signs whenever they are not crucial for the sake of clarity of our presentation. All logarithms are assumed to be base 2 . For $N>0$, we let $[N]=\{1, \ldots, N\}$.

\section{Proof of Theorem 1.3}

In this section we will prove Theorem 1.3. We first list several results that we will use. The first lemma is a classic result in graph theory.

- Lemma 2.1 (Kövari-Sós-Turán [10]). Let $G=(V, E)$ be a graph that does not contain a complete bipartite graph $K_{r, s}(1 \leq r \leq s)$ as a subgraph. Then $|E| \leq c_{s}|V|^{2-\frac{1}{r}}$, where $c_{s}>0$ is constant which only depends on $s$.

The next lemma we will use is a partitioning tool in discrete geometry known as simplicial partitions. We will use the dual version which requires the following definition. Let $\mathcal{L}$ be a set of lines in the plane. We say that a point $p$ crosses $\mathcal{L}$ if it is incident to at least one member of $\mathcal{L}$, but not incident to all members in $\mathcal{L}$.

- Lemma 2.2 (Matousek [12]). Let $\mathcal{L}$ be a set of $n$ lines in the plane and let $r$ be a parameter such that $1<r<n$. Then there is a partition on $\mathcal{L}=\mathcal{L}_{1} \cup \cdots \cup \mathcal{L}_{r}$ into $r$ parts, where $\frac{n}{2 r} \leq\left|\mathcal{L}_{i}\right| \leq \frac{2 n}{r}$, such that any point $p \in \mathbb{R}^{2}$ crosses at most $O(\sqrt{r})$ parts $\mathcal{L}_{i}$.

Proof of Theorem 1.3. Set $t \geq 2$. Let $P$ be a set of $m$ points in the plane and let $\mathcal{L}$ be a set of $n$ lines in the plane such that $(P, \mathcal{L})$ does not contain a subconfiguration isomorphic to $\left(P_{0}, \mathcal{L}_{a} \cup \mathcal{L}_{b}\right)$.

If $n \geq m^{2} / 100$, then $(1.1)$ implies that $|I(P, \mathcal{L})|=O(n)$ and we are done. Likewise, if $n \leq m^{\frac{t}{2 t-1}}$, then $(1.1)$ implies that $|I(P, \mathcal{L})|=O\left(m^{1+\frac{1}{6 t-3}}\right)$ and we are done. Therefore, let us assume $m^{\frac{t}{2 t-1}}<n<m^{2} / 100$. In what follows, we will show that $|I(P, \mathcal{L})|=O\left(m^{\frac{2 t-2}{3 t-2}} n^{\frac{2 t-1}{3 t-2}}\right)$. For sake of contradiction, suppose that $I(P, \mathcal{L}) \geq c m^{\frac{2 t-2}{3 t-2}} n^{\frac{2 t-1}{3 t-2}}$, where $c$ is a large constant depending on $t$ that will be determined later.

Set $r=\left\lceil 10 n^{\frac{4 t-2}{3 t-2}} / m^{\frac{2 t}{3 t-2}}\right\rceil$. Let us remark that $1<r<n / 10$ since we are assuming $m^{\frac{t}{2 t-1}}<n<m^{2} / 100$. We apply Lemma 2.2 with parameter $r$ to $\mathcal{L}$, and obtain the partition $\mathcal{L}=\mathcal{L}_{1} \cup \cdots \cup \mathcal{L}_{r}$ with the properties described above. Note that $\left|\mathcal{L}_{i}\right|>1$. Let $G$ be the incidence graph of $(P, \mathcal{L})$. For $p \in P$, consider the set of lines in $\mathcal{L}_{i}$. If $p$ is incident to exactly one line in $\mathcal{L}_{i}$, then delete the corresponding edge in the incidence graph $G$. After performing 
this operation between each point $p \in P$ and each part $\mathcal{L}_{i}$, by Lemma 2.2, we have deleted at most $c_{1} m \sqrt{r}$ edges in $G$, where $c_{1}$ is an absolute constant. By setting $c$ sufficiently large, we have

$$
c_{1} m \sqrt{r}=\sqrt{10} c_{1} m^{\frac{2 t-2}{3 t-2}} n^{\frac{2 t-1}{3 t-2}}<(c / 2) m^{\frac{2 t-2}{3 t-2}} n^{\frac{2 t-1}{3 t-2}} .
$$

Therefore, there are at least $(c / 2) m^{\frac{2 t-2}{3 t-2}} n^{\frac{2 t-1}{3 t-2}}$ edges remaining in $G$. By the pigeonhole principle, there is a part $\mathcal{L}_{i}$ such that the number of edges between $P$ and $\mathcal{L}_{i}$ in $G$ is at least

$$
\frac{c m^{\frac{2 t-2}{3 t-2}} n^{\frac{2 t-1}{3 t-2}}}{2 r}=\frac{c m^{\frac{4 t-2}{3 t-2}}}{20 n^{\frac{2 t-1}{3 t-2}}} .
$$

Hence, every point $p \in P$ has either 0 or at least 2 neighbors in $\mathcal{L}_{i}$ in $G$. We claim that $\left(P, \mathcal{L}_{i}\right)$ contains a subconfiguration isomorphic to $\left(P_{0}, \mathcal{L}_{a} \cup \mathcal{L}_{b}\right)$. To see this, let us construct a graph $H=\left(\mathcal{L}_{i}, E\right)$ as follows. Set $V(H)=\mathcal{L}_{i}$. Let $Q=\left\{q_{1}, \ldots, q_{w}\right\} \subset P$ be the set of points in $P$ that have at least two neighbors in $\mathcal{L}_{i}$ in the graph $G$. For $q_{j} \in Q$, consider the set of lines $\left\{\ell_{1}, \ldots, \ell_{s}\right\}$ from $\mathcal{L}_{i}$ incident to $q_{j}$, such that $\left\{\ell_{1}, \ldots, \ell_{s}\right\}$ appears in clockwise order. Then we define $E_{j} \subset\left(\begin{array}{c}\mathcal{L}_{i} \\ 2\end{array}\right)$ to be a matching on $\left\{\ell_{1}, \ldots, \ell_{s}\right\}$, where

$$
E_{j}= \begin{cases}\left\{\left(\ell_{1}, \ell_{2}\right),\left(\ell_{3}, \ell_{4}\right), \ldots,\left(\ell_{s-1}, \ell_{s}\right)\right\} & \text { if } s \text { is even } \\ \left\{\left(\ell_{1}, \ell_{2}\right),\left(\ell_{3}, \ell_{4}\right), \ldots,\left(\ell_{s-2}, \ell_{s-1}\right)\right\} & \text { if } s \text { is odd }\end{cases}
$$

Set $E(H)=E_{1} \cup E_{2} \cup \cdots \cup E_{w}$. Note that $E_{j}$ and $E_{k}$ are disjoint, since no two points are contained in two lines. Since $\left|E_{j}\right| \geq 1$, we have

$$
|E(H)| \geq \frac{c m^{\frac{4 t-2}{3 t-2}}}{60 n^{\frac{2 t-1}{3 t-2}}} .
$$

Since

$$
|V(H)|=\left|\mathcal{L}_{i}\right| \leq \frac{m^{\frac{2 t}{3 t-2}}}{5 n^{\frac{t}{3 t-2}}},
$$

this implies

$$
|E(H)| \geq \frac{c}{60 \cdot 25}(V(H))^{2-\frac{1}{t}}
$$

By setting $c=c(t)$ to be sufficiently large, Lemma 2.1 implies that $H$ contains a copy of $K_{t, t}$. Let $\mathcal{L}_{1}^{\prime}, \mathcal{L}_{2}^{\prime} \subset \mathcal{L}_{i}$ correspond to the vertices of this $K_{t, t}$ in $H$, and let $P^{\prime}=\left\{\ell_{1} \cap \ell_{2} \in P: \ell_{1} \in\right.$ $\left.\mathcal{L}_{1}^{\prime}, \ell_{2} \in \mathcal{L}_{2}^{\prime}\right\}$. We claim that $\left(P^{\prime}, \mathcal{L}_{1}^{\prime} \cup \mathcal{L}_{2}^{\prime}\right)$ is isomorphic to $\left(P_{0}, \mathcal{L}_{a} \cup \mathcal{L}_{b}\right)$. It suffices to show that $\left|P^{\prime}\right|=t^{2}$. For the sake of contradiction, suppose $p \in \ell_{1} \cap \ell_{2} \cap \ell_{3}$, where $\ell_{1}, \ell_{2} \in \mathcal{L}_{1}^{\prime}$ and $\ell_{3} \in \mathcal{L}_{2}^{\prime}$. This would imply $\left(\ell_{1}, \ell_{3}\right),\left(\ell_{2}, \ell_{3}\right) \in E_{j}$ for some $j$ which contradicts the fact that $E_{j} \subset\left(\begin{array}{c}\mathcal{L}_{i} \\ 2\end{array}\right)$ is a matching. Same argument follows if $\ell_{1} \in \mathcal{L}_{1}^{\prime}$ and $\ell_{2}, \ell_{3} \in \mathcal{L}_{2}^{\prime}$. This completes the proof of Theorem 1.3.

\section{Natural Grids}

Given a set of $n$ points $P$ and a set of $n$ lines $\mathcal{L}$ in the plane, if $|I(P, \mathcal{L})| \geq c n^{\frac{4}{3}-\frac{1}{9 k-6}}$, where $c$ is a sufficiently large constant depending on $k$, then Corollary 1.4 implies that there are two sets of $k$ lines such that each pair of them from different sets intersects at a unique point in $P$. Therefore, Theorem 1.6 follows by combining Theorem 1.3 with the following lemma. 


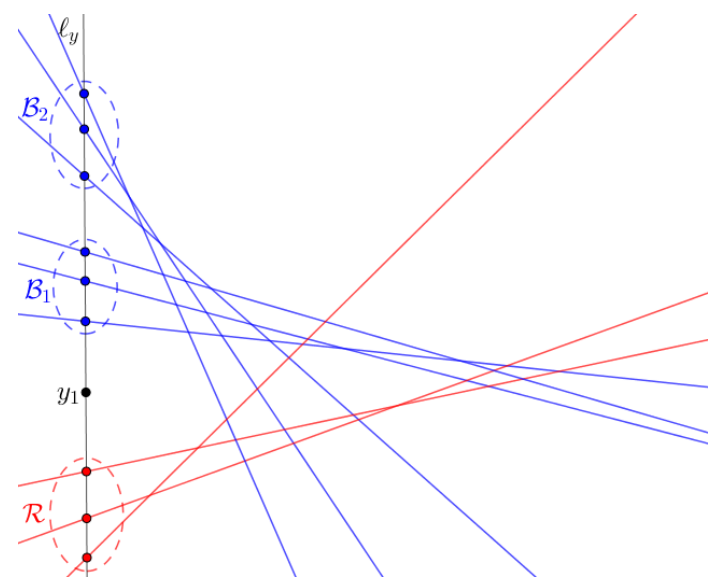

Figure 3 Sets $\mathcal{R}, \mathcal{B}_{1}, \mathcal{B}_{2}$ in the proof of Lemma 3.1.

- Lemma 3.1. There is a natural number $c$ such that the following holds. Let $\mathcal{B}$ be a set of $\mathrm{ct}^{2}$ blue lines in the plane, and let $\mathcal{R}$ be a set of $\mathrm{ct}^{2}$ red lines in the plane such that for $P=\left\{\ell_{1} \cap \ell_{2}: \ell_{1} \in \mathcal{B}, \ell_{2} \in \mathcal{R}\right\}$ we have $|P|=c^{2} t^{4}$. Then $(P, \mathcal{B} \cup \mathcal{R})$ contains a natural $t \times t$ grid.

To prove Lemma 3.1, we will need the following lemma which is an immediate consequence of Dilworth's Theorem.

- Lemma 3.2. For $n>0$, let $\mathcal{L}$ be a set of $n^{2}$ lines in the plane, such that no two members intersect the same point on the $y$-axis. Then there is a subset $\mathcal{L}^{\prime} \subset \mathcal{L}$ of size $n$ such that the intersection point of any two members in $\mathcal{L}^{\prime}$ lies to the left of the $y$-axis, or the intersection point of any two members in $\mathcal{L}^{\prime}$ lies to the right of the $y$-axis.

Proof. Let us order the elements in $\mathcal{L}=\left\{\ell_{1}, \ldots, \ell_{n^{2}}\right\}$ from bottom to top according to their $y$-intercept. By Dilworth's Theorem [5], $\mathcal{L}$ contains a subsequence of $n$ lines whose slopes are either increasing or decreasing. In the first case, all intersection points are to the left of the $y$-axis, and in the latter case, all intersection points are to the right of the $y$-axis.

Proof of Lemma 3.1. Let $(P, \mathcal{B} \cup \mathcal{R})$ be as described above, and let $\ell_{y}$ be the $y$-axis. Without loss of generality, we can assume that all lines in $\mathcal{B} \cup \mathcal{R}$ are not vertical, and the intersection point of any two lines in $\mathcal{B} \cup \mathcal{R}$ lies to the right of $\ell_{y}$. Moreover, we can assume that no two lines intersect at the same point on $\ell_{y}$.

We start by finding a point $y_{1} \in \ell_{y}$ such that at least $|\mathcal{B}| / 2$ blue lines in $\mathcal{B}$ intersect $\ell_{y}$ on one side of the point $y_{1}$ (along $\ell_{y}$ ) and at least $|\mathcal{R}| / 2$ red lines in $\mathcal{R}$ intersect $\ell_{y}$ on the other side. This can be done by sweeping the point $y_{1}$ along $\ell_{y}$ from bottom to top until $c t^{2} / 2$ lines of the first color, say red, intersect $\ell_{y}$ below $y_{1}$. We then have at least $c t^{2} / 2$ blue lines intersecting $\ell_{y}$ above $y_{1}$. Discard all red lines in $\mathcal{R}$ that intersect $\ell_{y}$ above $y_{1}$, and discard all blue lines in $\mathcal{B}$ that intersect $\ell_{y}$ below $y_{1}$. Hence, $|\mathcal{B}| \geq c t^{2} / 2$.

Set $s=\left\lfloor c t^{2} / 4\right\rfloor$. For the remaining lines in $\mathcal{B}$, let $\mathcal{B}=\left\{b_{1}, \ldots, b_{2 s}\right\}$, where the elements of $\mathcal{B}$ are ordered in the order they cross $\ell_{y}$, from bottom to top. We partition $\mathcal{B}=\mathcal{B}_{1} \cup \mathcal{B}_{2}$ into two parts, where $\mathcal{B}_{1}=\left\{b_{1}, \ldots, b_{s}\right\}$ and $\mathcal{B}_{2}=\left\{b_{s+1}, \ldots, b_{2 s}\right\}$. By applying an affine transformation, we can assume all lines in $\mathcal{R}$ have positive slope and all lines in $\mathcal{B}_{1} \cup \mathcal{B}_{2}$ have negative slope. See Figure 3. 


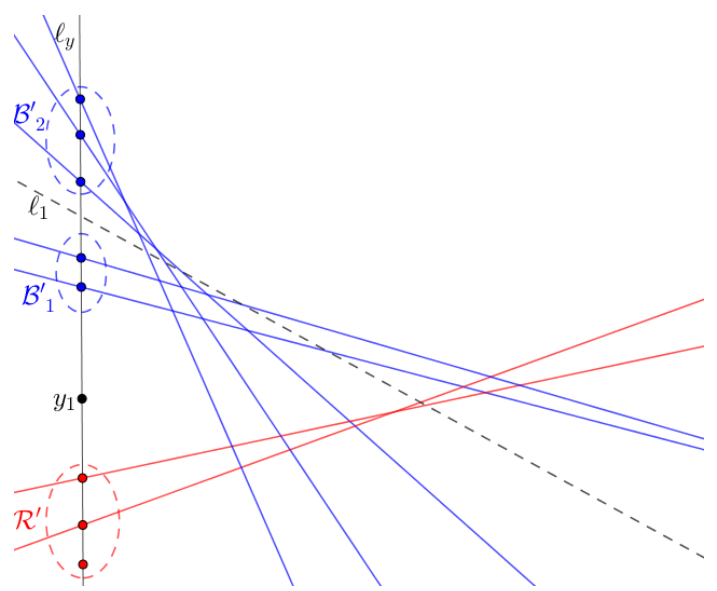

Figure 4 An example for the line $\ell_{1}$.

Let us define a 3-partite 3-uniform hypergraph $H=\left(\mathcal{R} \cup \mathcal{B}_{1} \cup \mathcal{B}_{2}, E\right)$, whose vertex parts are $\mathcal{R}, \mathcal{B}_{1}, \mathcal{B}_{2}$, and $\left(r, b_{i}, b_{j}\right) \in \mathcal{R} \times \mathcal{B}_{1} \times \mathcal{B}_{2}$ is an edge in $H$ if and only if the intersection point $p=b_{i} \cap b_{j}$ lies above the line $r$. Note, if $b_{i}$ and $b_{j}$ are parallel, then $\left(r, b_{i}, b_{j}\right) \notin E$. Then a result of Fox et al. on semi-algebraic hypergraphs implies the following (see also [3] and [9]).

- Lemma 3.3 (Fox et al. [8], Theorem 8.1). There exists a positive constant $\alpha$ such that the following holds. In the hypergraph above, there are subsets $\mathcal{R}^{\prime} \subseteq \mathcal{R}, \mathcal{B}_{1}^{\prime} \subseteq \mathcal{B}_{1}, \mathcal{B}_{2}^{\prime} \subseteq \mathcal{B}_{2}$, where $\left|\mathcal{R}^{\prime}\right| \geq \alpha|\mathcal{R}|,\left|\mathcal{B}_{1}^{\prime}\right| \geq \alpha\left|\mathcal{B}_{1}\right|,\left|\mathcal{B}_{2}^{\prime}\right| \geq \alpha\left|\mathcal{B}_{2}\right|$, such that either $\mathcal{R}^{\prime} \times \mathcal{B}_{1}^{\prime} \times \mathcal{B}_{2}^{\prime} \subseteq$ E, or $\left(\mathcal{R}^{\prime} \times \mathcal{B}_{1}^{\prime} \times \mathcal{B}_{2}^{\prime}\right) \cap E=\emptyset$

We apply Lemma 3.3 to $H$ and obtain subsets $\mathcal{R}^{\prime}, \mathcal{B}_{1}^{\prime}, \mathcal{B}_{2}^{\prime}$ with the properties described above. Without loss of generality, we can assume that $\mathcal{R}^{\prime} \times \mathcal{B}_{1}^{\prime} \times \mathcal{B}_{2}^{\prime} \subset E$, since a symmetric argument would follow otherwise. Let $\ell_{1}$ be a line in the plane such that the following holds.

1. The slope of $\ell_{1}$ is negative.

2. All intersection points between $\mathcal{R}^{\prime}$ and $\mathcal{B}^{\prime}{ }_{1}$ lie above $\ell_{1}$.

3. All intersection points between $\mathcal{R}^{\prime}$ and $\mathcal{B}^{\prime}{ }_{2}$ lie below $\ell_{1}$.

See Figure 4.

- Observation 3.4. Line $\ell_{1}$ defined above exists.

Proof. Let $U$ be the upper envelope of the arrangement $\bigcup_{\ell \in \mathcal{R}^{\prime}} \ell$, that is, $U$ is the closure of all points that lie on exactly one line of $\mathcal{R}^{\prime}$ and strictly above exactly the $\left|\mathcal{R}^{\prime}\right|-1$ lines in $\mathcal{R}^{\prime}$.

Let $P_{1}$ be the set of intersection points between the lines in $\mathcal{B}_{1}^{\prime}$ with $U$. Likewise, we define $P_{2}$ to be the set of intersection points between the lines in $\mathcal{B}_{2}^{\prime}$ with $U$. Since $U$ is $x$-monotone and convex the set $P_{2}$ lies to the left of the set $P_{1}$. Then the line $\ell_{1}$ that intersects $U$ between $P_{1}$ and $P_{2}$ and intersects $\ell_{y}$ between $\mathcal{B}_{1}^{\prime}$ and $\mathcal{B}_{2}^{\prime}$ satisfies the conditions above.

Now we apply Lemma 3.2 to $\mathcal{R}^{\prime}$ with respect to the line $\ell_{1}$, to obtain $\sqrt{\alpha c / 2} \cdot t$ members in $\mathcal{R}^{\prime}$ such that every pair of them intersects on one side of $\ell_{1}$. Discard all other members in $\mathcal{R}^{\prime}$. Without loss of generality, we can assume that all intersection points between any two members in $\mathcal{R}^{\prime}$ lie below $\ell_{1}$, since a symmetric argument would follow otherwise. We now discard the set $\mathcal{B}_{2}^{\prime}$.

Notice that the order in which the lines in $\mathcal{R}^{\prime}$ cross $b \in \mathcal{B}_{1}^{\prime}$ will be the same for any line $b \in \mathcal{B}_{1}^{\prime}$. Therefore, we order the elements in $\mathcal{R}^{\prime}=\left\{r_{1}, \ldots, r_{m}\right\}$ with respect to this ordering, from left to right, where $m=\lceil\sqrt{\alpha c / 2} \cdot t\rceil$. We define $\ell_{2}$ to be the line obtained by slightly perturbing the line $r_{\lfloor m / 2\rfloor}$ such that: 


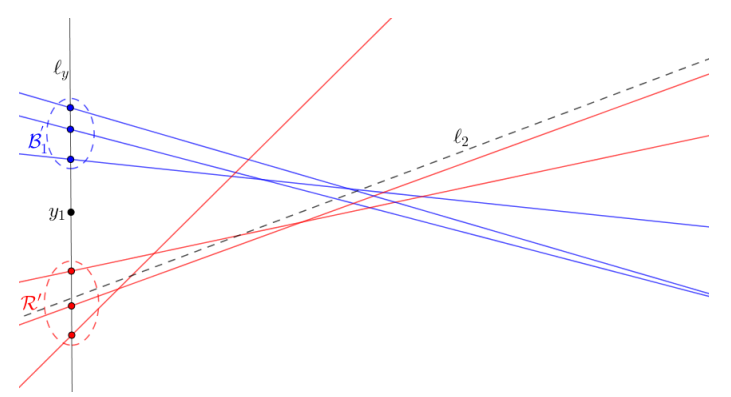

Figure 5 An example for the line $\ell_{2}$.

1. The slope of $\ell_{2}$ is positive.

2. All intersection points between $\mathcal{B}_{1}^{\prime}$ and $\left\{r_{1}, \ldots, r_{\lfloor m / 2\rfloor}\right\}$ lie above $\ell_{2}$.

3. All intersection points between $\mathcal{B}_{1}^{\prime}$ and $\left\{r_{\lfloor m / 2\rfloor+1}, \ldots, r_{m}\right\}$ lie below $\ell_{2}$.

See the Figure 5.

Finally, we apply Lemma 3.2 to $\mathcal{B}_{1}^{\prime}$ with respect to the line $\ell_{2}$, to obtain at least $\sqrt{\alpha c} \cdot t / 2$ members in $\mathcal{B}_{1}^{\prime}$ with the property that any two of them intersect on one side of $\ell_{2}$. Without loss of generality, we can assume that any two such lines intersect below $\ell_{2}$ since a symmetric argument would follow. Set $\mathcal{B}^{*} \subset \mathcal{B}_{1}^{\prime}$ to be these set of lines. Then $\mathcal{B}^{*} \cup\left\{r_{1}, \ldots, r_{\lfloor m / 2\rfloor}\right\}$ and their intersection points form a natural grid. By setting $c=c(t)$ to be sufficiently large, we obtain a natural $t \times t$ grid.

\section{Lower Bound Construction}

In this section, we will prove Theorem 1.5. First, let us recall the definitions of Sidon and $k$-fold Sidon sets.

Let $A$ be a finite set of positive integers. Then $A$ is a Sidon set if the sum of all pairs are distinct, that is, the equation $x+y=u+v$ has no solutions with $x, y, u, v \in A$, except for trivial solutions given by $u=x, y=v$ and $x=v, y=u$. We define $s(N)$ to be the size of the largest Sidon set $A \subset\{1, \ldots, N\}$. Erdős and Turán proved the following.

- Lemma 4.1 (See [7] and [14]). For $N>1$, we have $s(N)=\Theta(\sqrt{N})$.

Let us now consider a more general equation. Let $u_{1}, \ldots, u_{4}$ be integers such that $u_{1}+u_{2}+u_{3}+u_{4}=0$, and consider the equation

$u_{1} x_{1}+u_{2} x_{2}+u_{3} x_{3}+u_{4} x_{4}=0$.

We are interested in solutions to (4.1) with $x_{1}, x_{2}, x_{3}, x_{4} \in \mathbb{Z}$. Suppose $\left(x_{1}, x_{2}, x_{3}, x_{4}\right)=$ $\left(a_{1}, a_{2}, a_{3}, a_{4}\right)$ is an integer solution to (4.1). Let $d \leq 4$ be the number of distinct integers in the set $\left\{a_{1}, a_{2}, a_{3}, a_{4}\right\}$. Then we have a partition on the indices

$$
\{1,2,3,4\}=T_{1} \cup \cdots \cup T_{d},
$$

where $i$ and $j$ lie in the same part $T_{\nu}$ if and only if $x_{i}=x_{j}$. We call $\left(a_{1}, a_{2}, a_{3}, a_{4}\right)$ a trivial solution to (4.1) if

$$
\sum_{i \in T_{\nu}} u_{i}=0, \quad \nu=1, \ldots, d
$$

Otherwise, we will call $\left(a_{1}, a_{2}, a_{3}, a_{4}\right)$ a nontrivial solution to (4.1). 
In [11], Lazebnik and Verstraëte introduced $k$-fold Sidon sets which are defined as follows. Let $k$ be a positive integer. A set $A \subset \mathbb{N}$ is a $k$-fold Sidon set if each equation of the form

$$
u_{1} x_{1}+u_{2} x_{2}+u_{3} x_{3}+u_{4} x_{4}=0,
$$

where $\left|u_{i}\right| \leq k$ and $u_{1}+\cdots+u_{4}=0$, has no nontrivial solutions with $x_{1}, x_{2}, x_{3}, x_{4} \in A$. Let $r(k, N)$ be the size of the largest $k$-fold Sidon set $A \subset\{1, \ldots, N\}$.

- Lemma 4.2. There is an infinite sequence $1=a_{1}<a_{2}<\cdots$ of integers such that

$$
a_{m} \leq 2^{8} k^{4} m^{3},
$$

and the system of equations (4.2) has no nontrivial solutions in the set $A=\left\{a_{1}, a_{2}, \ldots\right\}$. In particular, for integers $N>k^{4} \geq 1$, we have $r(k, N) \geq c k^{-4 / 3} N^{1 / 3}$, where $c$ is a positive constant.

The proof of Lemma 4.2 is a slight modification of the proof of Theorem 2.1 in [14]. For the sake of completeness, we include the proof here.

Proof. We put $a_{1}=1$ and define $a_{m}$ recursively. Given $a_{1}, \ldots, a_{m-1}$, let $a_{m}$ be the smallest positive integer satisfying

$$
a_{m} \neq-\left(\sum_{i \in S} u_{i}\right)^{-1} \sum_{1 \leq i \leq 4, i \notin S} u_{i} x_{i}
$$

for every choice $u_{i}$ such that $\left|u_{i}\right| \leq k$, for every set $S \subset\{1, \ldots, 4\}$ of subscripts such that $\left(\sum_{i \in S} u_{i}\right) \neq 0$, and for every choice of $x_{i} \in\left\{a_{1}, \ldots, a_{m-1}\right\}$, where $i \notin S$. For a fixed $S$ with $|S|=j$, this excludes $(m-1)^{4-j}$ numbers. Since $\left|u_{i}\right| \leq k$, the total number of excluded integers is at most

$$
(2 k+1)^{4} \sum_{j=1}^{3}\left(\begin{array}{l}
4 \\
j
\end{array}\right)(m-1)^{4-j}=(2 k+1)^{4}\left(m^{4}-(m-1)^{4}-1\right)<2^{8} k^{4} m^{3} .
$$

Consequently, we can extend our set by an integer $a_{m} \leq 2^{8} k^{4} m^{3}$. This will automatically be different from from $a_{1}, \ldots, a_{m-1}$, since putting $x_{i}=a_{j}$ for all $i \notin S$ in (4.3) we get $a_{m} \neq a_{j}$. It will also satisfy $a_{m}>a_{m-1}$ by minimal choice of $a_{m-1}$.

We show that the system of equations (4.2) has no nontrivial solutions in the set $\left\{a_{1}, \ldots, a_{m}\right\}$. We use induction on $m$. The statement is obviously true for $m=1$. We establish it for $m$ assuming for $m-1$. Suppose that there is a nontrivial solution $\left(x_{1}, x_{2}, x_{3}, x_{4}\right)$ to (4.2) for some $u_{1}, u_{2}, u_{3}, u_{4}$ with the properties described above. Let $S$ denote the set of those subscripts for which $x_{i}=a_{m}$. If $\sum_{i \in S} u_{i} \neq 0$, then this contradicts (4.3). If $\sum_{i \in S} u_{i}=0$, then by replacing each occurrence of $a_{m}$ by $a_{1}$, we get another nontrivial solution, which contradicts the induction hypothesis.

For more problems and results on Sidon sets and $k$-fold Sidon sets, we refer the interested reader to $[11,14,4]$.

We are now ready to prove Theorem 1.5.

Proof of Theorem 1.5. We start by applying Lemma 4.1 to obtain a Sidon set $M \subset\left[n^{1 / 7}\right]$, such that $|M|=\Theta\left(n^{1 / 14}\right)$. We then apply Lemma 4.2 with $k=n^{1 / 7}$ and $N=\frac{1}{4} n^{11 / 14}$, to obtain a $k$-fold Sidon set $A \subset[N]$ such that

$$
|A| \geq c n^{1 / 14}
$$

where $c$ is defined in Lemma 4.2. Without loss of generality, let us assume $|A|=c n^{1 / 14}$. 
Let $P=\left\{(i, j) \in \mathbb{Z}^{2}: i \in A, 1 \leq j \leq n^{13 / 14}\right\}$, and let $\mathcal{L}$ be the family of lines in the plane of the form $y=m x+b$, where $m \in M$ and $b$ is an integer such that $1 \leq b \leq n^{13 / 14} / 2$.

Hence, we have

$$
\begin{aligned}
& |P|=|A| \cdot n^{13 / 14}=\Theta(n), \\
& |\mathcal{L}|=|M| \cdot \frac{n^{13 / 14}}{2}=\Theta(n) .
\end{aligned}
$$

Notice that each line in $\mathcal{L}$ has exactly $|A|=c n^{1 / 14}$ points from $P$ since $1 \leq b \leq n^{13 / 14} / 2$. Therefore,

$$
|I(P, \mathcal{L})|=|\mathcal{L}||A|=\Theta\left(n^{1+1 / 14}\right) .
$$

$\triangleright$ Claim 4.3. There are no four distinct lines $\ell_{1}, \ell_{2}, \ell_{3}, \ell_{4} \in \mathcal{L}$ and four distinct points $p_{1}, p_{2}, p_{3}, p_{4} \in P$ such that $\ell_{1} \cap \ell_{2}=p_{1}, \ell_{2} \cap \ell_{3}=p_{2}, \ell_{3} \cap \ell_{4}=p_{3}, \ell_{4} \cap \ell_{1}=p_{4}$.

Proof. For the sake of contradiction, suppose there are four lines $\ell_{1}, \ell_{2}, \ell_{3}, \ell_{4}$ and four points $p_{1}, p_{2}, p_{3}, p_{4}$ with the properties described above. Let $\ell_{i}=m_{i} x+b_{i}$ and let $p_{i}=\left(x_{i}, y_{i}\right)$. Therefore,

$$
\begin{aligned}
& \ell_{1} \cap \ell_{2}=p_{1}=\left(x_{1}, y_{1}\right), \\
& \ell_{2} \cap \ell_{3}=p_{2}=\left(x_{2}, y_{2}\right), \\
& \ell_{3} \cap \ell_{4}=p_{3}=\left(x_{3}, y_{3}\right), \\
& \ell_{4} \cap \ell_{1}=p_{4}=\left(x_{4}, y_{4}\right) .
\end{aligned}
$$

Hence,

$$
\begin{aligned}
& p_{1} \in \ell_{1}, \ell_{2} \quad \Longrightarrow\left(m_{1}-m_{2}\right) x_{1}+b_{1}-b_{2}=0, \\
& p_{2} \in \ell_{2}, \ell_{3} \quad \Longrightarrow\left(m_{2}-m_{3}\right) x_{2}+b_{2}-b_{3}=0, \\
& p_{3} \in \ell_{3}, \ell_{4} \quad \Longrightarrow\left(m_{3}-m_{4}\right) x_{3}+b_{3}-b_{4}=0, \\
& p_{4} \in \ell_{4}, \ell_{1} \quad \Longrightarrow\left(m_{4}-m_{1}\right) x_{4}+b_{4}-b_{1}=0 .
\end{aligned}
$$

By summing up the four equations above, we get

$$
\left(m_{1}-m_{2}\right) x_{1}+\left(m_{2}-m_{3}\right) x_{2}+\left(m_{3}-m_{4}\right) x_{3}+\left(m_{4}-m_{1}\right) x_{4}=0 .
$$

By setting $u_{1}=m_{1}-m_{2}, u_{2}=m_{2}-m_{3}, u_{3}=m_{3}-m_{4}, u_{4}=m_{4}-m_{1}$, we get

$$
u_{1} x_{1}+u_{1} x_{2}+u_{3} x_{3}+u_{4} x_{4}=0,
$$

where $u_{1}+u_{2}+u_{3}+u_{4}=0$ and $\left|u_{i}\right| \leq n^{1 / 7}$. Since $x_{1}, \ldots, x_{4} \in A,\left(x_{1}, x_{2}, x_{3}, x_{4}\right)$ must be a trivial solution to (4.4). The proof now falls into the following cases, and let us note that no line in $\mathcal{L}$ is vertical.

Case 1. Suppose $x_{1}=x_{2}=x_{3}=x_{4}$. Then $\ell_{i}$ is vertical and we have a contradiction.

Case 2. Suppose $x_{1}=x_{2}=x_{3} \neq x_{4}$ and $u_{1}+u_{2}+u_{3}=0$ and $u_{4}=0$. Then $\ell_{1}$ and $\ell_{4}$ have the same slope which is a contradiction. The same argument follows if $x_{1}=x_{2}=x_{4} \neq x_{3}$, $x_{1}=x_{3}=x_{4} \neq x_{2}$, or $x_{2}=x_{3}=x_{4} \neq x_{1}$. 
Case 3. Suppose $x_{1}=x_{2} \neq x_{3}=x_{4}, u_{1}+u_{2}=0$, and $u_{3}+u_{4}=0$. Since $p_{1}, p_{2} \in \ell_{2}$ and $x_{1}=x_{2}$, this implies that $\ell_{2}$ is vertical which is a contradiction. A similar argument follows if $x_{1}=x_{4} \neq x_{2}=x_{3}, u_{1}+u_{4}=0$, and $u_{2}+u_{3}=0$.

Case 4. Suppose $x_{1}=x_{3} \neq x_{2}=x_{4}, u_{1}+u_{3}=0$, and $u_{2}+u_{4}=0$. Then $u_{1}+u_{3}=0$ implies that $m_{1}+m_{3}=m_{2}+m_{4}$. Since $M$ is a Sidon set, we have either $m_{1}=m_{2}$ and $m_{3}=m_{4}$ or $m_{1}=m_{4}$ and $m_{2}=m_{3}$. The first case implies that $\ell_{1}$ and $\ell_{2}$ are parallel which is a contradiction, and the second case implies that $\ell_{2}$ and $\ell_{3}$ are parallel, which is again a contradiction.

This completes the proof of Theorem 1.5.

\section{$5 \quad$ Concluding Remarks}

- An old result of Erdős states that every $n$-vertex graph that does not contain a cycle of length $2 k$, has $O_{k}\left(n^{1+1 / k}\right)$ edges. It is known that this bound is tight when $k=2,3$, and 5 , but it is a long standing open problem in extremal graph theory to decide whether or not this upper bound can be improved for other values of $k$. Hence, Erdös's upper bound of $O\left(n^{5 / 4}\right)$ when $k=4$ implies Theorem 1.3 when $t=2$ and $m=n$. It would be interesting to see if one can improve the upper bound in Theorem 1.3 when $t=2$. For more problems on cycles in graphs, see [17].

- The proof of Lemma 3.1 is similar to the proof of the main result in [1]. The main difference is that we use the result of Fox et al. [8] instead of the Ham-Sandwich Theorem. We also note that a similar result was established by Dujmović and Langerman (see Theorem 6 in $[6])$.

\section{References}

1 Boris Aronov, Paul Erdős, Wayne Goddard, Daniel Kleitman, Michael Klugerman, János Pach, and Leonard J. Schulman. Crossing families. Combinatorica, 14(2):127-134, 1994.

2 Peter Brass, William O.J. Moser, and János Pach. Research problems in discrete geometry. Springer Science \& Business Media, 2006.

3 Boris Bukh and Alfredo Hubard. Space crossing numbers. Combin. Probab. Comput., 21(3):358373, 2012.

4 Javier Cilleruelo and Craig Timmons. k-Fold Sidon Sets. Electron. J. Combin., 21(4):P4-12, 2014.

5 Robert P Dilworth. A decomposition theorem for partially ordered sets. Ann. of Math., pages 161-166, 1950.

6 Vida Dujmović and Stefan Langerman. A Center Transversal Theorem for Hyperplanes and Applications to Graph Drawing. Discrete Comput. Geom., 49(1):74-88, January 2013. doi:10.1007/s00454-012-9464-y.

7 Paul Erdös and Pál Turán. On a problem of Sidon in additive number theory, and on some related problems. J. Lond. Math. Soc. (2), 1(4):212-215, 1941.

8 Jacob Fox, Mikhail Gromov, Vincent Lafforgue, Assaf Naor, and János Pach. Overlap properties of geometric expanders. J. Reine Angew. Math., 2012(671):49-83, 2012.

9 Jacob Fox, János Pach, and Andrew Suk. A polynomial regularity lemma for semialgebraic hypergraphs and its applications in geometry and property testing. SIAM J. Comput., 45(6):2199-2223, 2016.

10 Tamás Kovári, Vera Sós, and Pál Turán. On a problem of K. Zarankiewicz. 3(1):50-57, 1954.

11 Felix Lazebnik and Jacques Verstraëte. On hypergraphs of girth five. Electron. J. Combin., 10:1-25, 2003.

12 Jiří Matoušek. Efficient partition trees. Discrete Comput. Geom., 8(3):315-334, 1992. 
13 János Pach and Pankaj K Agarwal. Combinatorial geometry, volume 37. John Wiley \& Sons, 2011.

14 Imre Z Ruzsa. Solving a linear equation in a set of integers I. Acta Arith., 65(3):259-282, 1993.

15 József Solymosi. Dense arrangements are locally very dense I. SIAM J. Discrete Math., 20(3):623-627, 2006.

16 Endre Szemerédi and William T. Trotter. Extremal problems in discrete geometry. Combinatorica, 3(3-4):381-392, 1983.

17 Jacques Verstraëte. Extremal problems for cycles in graphs. In Recent Trends in Combinatorics, pages 83-116. Springer, 2016. 